\title{
OPEN Investigating the association of testosterone with survival in men and women using a Mendelian randomization study in the UK Biobank
}

\author{
C. M. Schooling ${ }^{1,2 \bowtie ~} \&$ J. V. Zhao ${ }^{1}$
}

Life expectancy in the developed West is currently stagnated and remains shorter in men than women. Well-established evolutionary biology theory suggests lifespan trades-off against reproductive success, possibly sex-specifically. We examined whether a key driver of reproductive success, testosterone, affected survival using a Mendelian randomization longevity study in the UK Biobank to obtain unbiased estimates, along with control exposures. We applied published genetic instruments for testosterone to obtain inverse variance weighted estimates of associations with survival to (i.e., age at) recruitment, in 167,020 men and 194,174 women. We similarly obtained estimates for a positive control (smoking initiation), and a negative control (absorbate), a marker of vitamin $C$ metabolism. Testosterone was associated with poorer survival ( 0.10 years younger at recruitment per effect size of testosterone, $95 \%$ confidence interval ( $\mathrm{Cl}) 0.004$ to 0.20 ). As expected, smoking initiation was also associated with poorer survival $(0.37$ years younger, $95 \% \mathrm{Cl} 0.25$ to 0.50$)$, but not absorbate ( 0.01 years younger, $95 \% \mathrm{Cl}-0.09$ to 0.11 ). Several aspects of a healthy lifestyle (low animal fat diet) and several widely used medications (statins, metformin, dexamethasone and possibly aspirin) may modulate testosterone. Explicitly designing interventions sex-specifically based on these insights might help address stagnating life expectancy and sexual disparities.

Sexual dimorphism in lifespan is widely evident, including among humans, contemporaneously and historically ${ }^{1,2}$. Many reasons have been advanced for shorter life expectancy in men than women, from gendered health and health care seeking behavior ${ }^{3}$ through sex-specific nutrient metabolism and steroid hormones ${ }^{4}$ to sex-specific sexual selection pressures ${ }^{5,6}$, but few of these have been explicitly exploited as a means of promoting healthy aging in both sexes. As the long-term trend of increasing life expectancy in the West now appears to be stagnating ${ }^{7}$, reconsidering overlooked targets and their implications for the design of interventions might bear consideration.

An obvious modifiable difference between men and women is levels of sex hormones. Estrogen has been intensively investigated as a protective factor that might explain differences in life expectancy by sex. Observational evidence concerning benefits of exogenous estrogen in women was very promising ${ }^{8}$. Large-scale trials of estrogen in women and men were stopped early for harm or lack of benefit ${ }^{9}$. Discrepant findings in trials compared to observational studies have been ascribed to overlooked confounding by socio-economic position in the observational studies ${ }^{9}$. Promising observational evidence exists for survival benefits of endogenous testosterone in women ${ }^{10-12}$ and men $^{13,14}$, although some inconsistencies exist ${ }^{15-17}$. Testosterone in men falls with aging and ill-health ${ }^{18-20}$, likely generating residual confounding, as health status is difficult to adjust for comprehensively. A trial of the cardiovascular safety of exogenous testosterone in women has been successfully conducted ${ }^{21}$. A small trial of exogenous testosterone in frail older men was stopped early because of adverse events in the testosterone $\operatorname{arm}^{22}$, but is difficult to interpret. A larger trial of exogenous testosterone on cardiovascular disease is now underway in men $^{23}$. Theoretically, the expectation for effects of testosterone on health might be in a similar direction to those found for estrogen because of the well-established Darwinian evolutionary biology trade-off between reproduction, and its drivers, on the one hand, and survival, on the other hand ${ }^{24-26}$.

${ }^{1}$ School of Public Health, Li Ka Shing, Faculty of Medicine, The University of Hong Kong, 1/F, Patrick Manson Building (North Wing), 7 Sassoon Road, Pokfulam, Hong Kong, China. ${ }^{2}$ Graduate School of Public Health and Health Policy, City University of New York, New York, NY, USA. email: cms1@hku.hk 


\begin{tabular}{|l|l|l|l|l|}
\hline Exposure type & Exposure & $\begin{array}{l}\text { Difference in age at } \\
\text { recruitment (years) }\end{array}$ & 95\% CI & $\boldsymbol{p}$ value \\
\hline Positive control & Smoking initiation compared to not & -0.37 & -0.50 to -0.25 & $5.2 \mathrm{e}-9$ \\
\hline Negative control & Absorbate (rs33972313) & -0.01 & -0.11 to 0.09 & 0.79 \\
\hline Exposure & Testosterone (standard deviation) & -0.10 & -0.20 to -0.004 & 0.04 \\
\hline
\end{tabular}

Table 1. Differences in age at recruitment to the UK Biobank for published genetic predictors of testosterone $^{28}$, smoking ${ }^{29}$, and vitamin C (absorbate) ${ }^{30}$ using Mendelian Randomization inverse variance weighted estimates.

Here we conducted a Mendelian randomization (MR) study of the effects of testosterone on survival in men and women. MR studies take advantage of the random allocation of genetic material at conception to obtain less confounded estimates ${ }^{27}$. Specifically, MR studies use genetic variants as proxies of the exposure, and compare outcomes according to genetically predicted differences in exposure, i.e., use an instrumental variable design with genetic instruments. We used existing genetic predictors of testosterone and the control exposures ${ }^{28-30}$ and applied them to a study of longevity. Longevity studies compare characteristics of survivors to older ages with characteristics of younger people because lifetime harmful factors inevitably become less common with increasing age ${ }^{31}$. In contrast, observational prospective cohort studies comparing mortality incidence after recruitment are open to selection bias from any deaths prior to recruitment having already depleted the susceptibles ${ }^{32}$ which may attenuate estimates even to the extent of suggesting no benefit of proven healthy interventions ${ }^{33}$. As such, longevity studies, take advantage of the changing structure of risk factors with age to obtain estimates of effects on mortality free from selection bias ${ }^{34}$. In addition, as this is a hypothesis driven study, we also included positive and negative control exposures. Smoking initiation was used as a positive control outcome because smoking is very well-known to be addictive and to substantially reduce survival to old age $\mathrm{e}^{35}$. A marker of vitamin C metabolism, absorbate, was used as a negative control outcome because vitamin $\mathrm{C}$ is no longer thought to affect health ${ }^{36}$.

\section{Results}

In total 167,020 men and 194,174 women from the UK Biobank were included. Almost all the genetic variants used as instruments for the exposures (testosterone (125 variants in men and 254 variants in women), smoking initiation (361) and absorbate (1)) were available for the outcome. Two variants for smoking initiation were replaced by highly correlated $\left(\mathrm{r}^{2}>0.9\right)$ variants ( $\mathrm{rs} 2587507$ replaced by rs745571, and rs2359180 by rs768576) obtained from LDlink (https://ldlink.nci.nih.gov/). The average F-statistic for testosterone was 128.6 in men and 83.6 in women, and for smoking initiation was 44.9. As expected, smoking initiation was associated with younger age at recruitment (Table 1), corresponding to poorer survival. Similarly, as expected absorbate was unrelated to age at recruitment (Table 1). Overall, testosterone was associated with younger age at recruitment, i.e., poorer survival (Table 1), with slightly larger estimates in men than women (Table S1). Estimates were similar in sensitivity analysis using age at recruitment as a categorical variable (age 60 years or less) (Table S2) and using sensitivity analysis (Table S1).

\section{Discussion}

Despite observational studies suggesting that testosterone might promote longer life ${ }^{10-14}$, our study with greater robustness to confounding and selection bias, consistent with theoretical expectations from evolutionary biology $y^{24,25}$, suggests that testosterone reduces survival. Our study also showed smoking initiation reduced survival as would be expected ${ }^{35}$.

Our findings differ from previous observational studies which suggest that endogenous testosterone might improve health and lifespan ${ }^{10-14}$. However, these studies are open to both confounding by health status ${ }^{18,19}$ and selection bias from inevitably only recruiting survivors ${ }^{32}$. Our findings are more consistent with previous MR studies suggesting exogenous testosterone increases cardiovascular disease ${ }^{37,38}$, and corresponding warnings from regulators (https://www.fda.gov/drugs/drug-safety-and-availability/fda-drug-safety-communication-fdacautions-about-using-testosterone-products-low-testosterone-due). Our findings are also consistent with the observation that in one of the few primate species with no age or sex difference in testosterone levels, there is also no sex difference in age-dependant survival.

Several potential mechanisms could underlie our findings. Experimental studies suggest testosterone increases coronary plaque volume $\mathrm{e}^{39}$ and coagulation ${ }^{40}$ while impairing endothelial function ${ }^{41-43}$. Testosterone increases vulnerability to hormone related cancers, including prostate cancer ${ }^{28}$, breast cancer ${ }^{28}$ and endometrial cancer ${ }^{28}$. Testosterone is also increasingly acknowledged to be an immune-suppressant ${ }^{44}$, potentially increasing susceptibility to cancer $^{45}$, as well as to infectious diseases ${ }^{44}$, such as coronavirus disease 19 . Testosterone may also induce impulsive behaviour ${ }^{46}$.

Relevance to interventions. Given the importance of testosterone to reproductive success, testosterone is likely responsive to environmental indicators of suitable conditions for procreation, and hence a modifiable target of intervention. Serendipitously, many aspects of a healthy lifestyle and several widely used interventions reduce testosterone. A healthy diet, i.e., low-fat, high fiber, high soy, and low animal fat reduces testosterone in men $^{47-49}$ and women ${ }^{50}$. Exercise and weight loss reduce testosterone in women ${ }^{51-53}$, but possibly less so in men $^{54,55}$, although severe caloric restriction ( $50 \%$ energy requirements) reduces testosterone in men $^{56}$. One of the 
most effective medications for preventing and treating cardiovascular disease, i.e., statins, reduces testosterone ${ }^{57}$. The first-line treatment for diabetes, metformin, may reduce testosterone in women ${ }^{58}$, but not men ${ }^{59}$. Aspirin may also reduce testosterone ${ }^{60}$. Another essential medicine, dexamethasone reduces testosterone ${ }^{61,62}$. As such, many current interventions to promote healthy aging do happen to reduce testosterone, particularly in women. Explicitly, searching sex-specifically for interventions based on this insight might facilitate the search for new means of promoting healthy aging by identifying at an early stage any potential interventions that are likely to be unsuccessful, and where interventions should be sex-specific.

Limitations. Although this study used an innovative approach to generate an unbiased assessment of the effect of testosterone on survival, it has several limitations. First, MR has stringent assumptions including that the genetic instruments predict the exposures, hence our use of published instruments ${ }^{28-30}$, that the instruments are independent of exposure outcome confounders, which stems from the use of genetic instruments not related to confounders such as health status, lifestyle and socioeconomic position, and that the instruments only affect the outcome via the exposure, hence our use of sensitivity analysis and control exposures. Second, we assumed a linear relation of survival with age when it is exponential. However, at the relatively young age (mean age 57 years, intended recruitment age 40 to 69 years) considered here such an approximation is less of an issue than in old age. A sensitivity analysis using survival to age 60 years as the outcome gave a similar interpretation for genetically predicted testosterone (Table S2). Third, the samples used for exposures and outcome overlap. However, that would be expected to bias towards the null ${ }^{63}$, so our estimates of associations may be conservative. Moreover, the potential bias is less of concern as we did not use weak instruments to predict exposures ${ }^{63}$. Fourth, our study gives lifetime effects of endogenous testosterone up to age $\sim 57$ years rather than the effect of an intervention. However, the estimates for testosterone can be contextualized by the estimates for smoking. Fifth, longevity studies have been criticized for not taking account of cohort effects ${ }^{34}$. However, there is no reason to think that genetics are susceptible to cohort effects in the UK Biobank. Sixth, canalization, i.e., compensation for genetic effects might exist. However, this would likely bias towards the null. Seventh, the UK Biobank is not a population representative study. However, we used a study of longevity rather than a traditional prospective cohort study to avoid selection bias. Eighth, the relatively young age at recruitment, and correspondingly high survival means this study lacks power, so we focused on major determinants of healthy aging, but were not able to ascertain if the effects of testosterone were greater in men than women. Ninth, we were unable to replicate this study using a genome wide association study of life span or parental life span. Such studies typically do not include the $\mathrm{X}$ chromosome, which is important for testosterone. They also typically recruit in middle-age, i.e., recruit survivors, instead of taking account of those who have already died before recruitment, so are open to selection bias. Tenth, the effects of testosterone may vary by age. However, causes are generally expected to act consistently, although they may not be relevant in all situations ${ }^{64}$. Testosterone in men falls with age ${ }^{20}$, so these findings could be relatively less relevant to older men, but absolute risk of death increases with age.

\section{Conclusions}

Consistent with well-established evolutionary biology theory ${ }^{24-26}$ testosterone appeared to reduce survival. Serendipitously, many existing strategies for promoting healthy aging reduce testosterone, particularly in women. Explicitly using an established theory as a guide might facilitate the search for new interventions, and draws attention to the importance of sex-specific interventions.

\section{Methods}

Data sources. The UK Biobank recruited half a million people intended to be aged 40 to 69 years from across Great Britain in 2006-1065. Average age is about 57 years and just over half the participants are women. We extracted overall and sex-specific genetic associations with age at recruitment from publicly available UK Biobank summary statistics in white British (http://www.nealelab.is/uk-biobank). These specific summary statistics were generated from linear regression adjusted for sex and the first 20 principal components, after excluding poor quality samples. Where relevant genetic information was not available from these summary statistics, we similarly generated corresponding information from the UK Biobank individual data.

Exposures. We used published sex-specific genetic predictors of testosterone (125 variants for men and 254 for women $)^{28}$. We used published genetic predictors of smoking initiation (361 variants) $)^{29}$, and of vitamin C metabolism (1 variant $)^{30}$.

Outcome. In this study, we used age at recruitment to the UK Biobank as a proxy for survival, as there is no particular reason, apart from prior death (i.e., lack of survival), why genetics should generate differences in willingness to participate by age. So, harmful exposures would be expected to be associated with younger age at recruitment.

Statistical analysis. We obtained the F-statistics for the genetic instruments using an established approximation (square of genetic variant on exposure divided by its variance) ${ }^{66}$. As an F-statistic of 10 or less is usually taken as indicating a weak instrument. We aligned genetic associations on the same allele for exposures and outcome. We included palindromic and non-biallelic genetic variants because the genetic associations with exposures and outcome were obtained in the same study or have been used in the same study. We used proxies, as necessary. We meta-analyzed genetic variant specific Wald estimates (ratio of genetic variant on outcome to genetic variant on exposure) using inverse variance weighting (IVW) with multiplicative random effects. 
IVW estimates assume balance pleiotropy ${ }^{67}$. We used estimates with different assumptions as sensitivity analysis. The weighted median has a majority valid assumption and assumes $>50 \%$ of the genetic variants are valid instruments, while the contamination mixture method has a contrasting plurality valid assumption ${ }^{67}$. MR-Egger detects pleiotropic effects acting on the outcome other than via the exposure, but assumes that the genetic instruments do not act via confounders of exposure on outcome ${ }^{66}$. To avoid pleiotropic effects of sex hormone binding globulin, we used established genetic predictors of bio-available testosterone in men and total testosterone in women, as previously ${ }^{68}$. Bio-available testosterone may also be a more sensitive indicator that total testosterone of active testosterone in men ${ }^{69}$. Where necessary we combined estimates for men and women using IVW metaanalysis.

Given this is a hypothesis driven study, with a positive and a negative control, we used a statistical significance level of 0.05. All statistical analysis was conducted using R version 3.6.1 (The R Foundation for Statistical Computing, Vienna, Austria). The MendelianRandomization R package was used for the MR estimates and the metafor R package for meta-analysis. Estimates of genetic associations were taken from publicly available UK Biobank summary statistics, except for the unavailable associations which were based on individual level genetic associations from the UK Biobank obtained under application \#42468. All UK Biobank data was collected with fully informed consent.

\section{Data availability}

JVZ and CMS had full access to the data and take full responsibility for the results.

Received: 12 February 2021; Accepted: 9 June 2021

Published online: 07 July 2021

\section{References}

1. Lemaitre, J. F. et al. Sex differences in adult lifespan and aging rates of mortality across wild mammals. Proc. Natl. Acad. Sci. U. S. A. 117(15), 8546-8553 (2020).

2. Zarulli, V. et al. Women live longer than men even during severe famines and epidemics. Proc. Natl. Acad. Sci. U. S. A. 115(4), E832-e840 (2018).

3. Bartz, D. et al. Clinical advances in sex- and gender-informed medicine to improve the health of all: A review. JAMA Intern. Med. 180, $574(2020)$.

4. Regan, J. C. \& Partridge, L. Gender and longevity: Why do men die earlier than women? Comparative and experimental evidence. Best Pract. Res. Clin. Endocrinol. Metab. 27(4), 467-479 (2013).

5. Schooling, C. M. Could androgens be relevant to partly explain why men have lower life expectancy than women?. J. Epidemiol. Community Health 70(4), 324-328 (2016).

6. Gardner, E. J. et al. Sex-biased reduction in reproductive success drives selective constraint on human genes. bioRxiv https://doi. org/10.1101/20200526116111 (2020).

7. Lopez, A. D. \& Adair, T. Is the long-term decline in cardiovascular-disease mortality in high-income countries over? Evidence from national vital statistics. Int. J. Epidemiol. 48(6), 1815-1823 (2019).

8. Stampfer, M. J. \& Colditz, G. A. Estrogen replacement therapy and coronary heart disease: A quantitative assessment of the epidemiologic evidence. Prev. Med. 20(1), 47-63 (1991).

9. Petitti, D. Commentary: Hormone replacement therapy and coronary heart disease: Four lessons. Int. J. Epidemiol. 33(3), 461-463 (2004).

10. Schaffrath, G. et al. Association of sex hormones with incident 10-year cardiovascular disease and mortality in women. Maturitas 82(4), 424-430 (2015).

11. Benn, M. et al. Extreme concentrations of endogenous sex hormones, ischemic heart disease, and death in women. Arterioscler. Thromb. Vasc. Biol. 35(2), 471-477 (2015).

12. Sievers, C. et al. Low testosterone levels predict all-cause mortality and cardiovascular events in women: A prospective cohort study in German primary care patients. Eur. J. Endocrinol. 163(4), 699-708 (2010).

13. Araujo, A. B. et al. Clinical review: Endogenous testosterone and mortality in men: a systematic review and meta-analysis. J. Clin. Endocrinol. Metab. 96(10), 3007-3019 (2011).

14. Bentmar Holgersson, M., Landgren, F., Rylander, L. \& Lundberg, G. Y. Mortality is linked to low serum testosterone levels in younger and middle-aged men. Eur. Urol. 71(6), 991-992 (2017).

15. Holmboe, S. A. et al. The association of reproductive hormone levels and all-cause, cancer, and cardiovascular disease mortality in men. J. Clin. Endocrinol. Metab. 100(12), 4472-4480 (2015).

16. Phillips, A. C., Gale, C. R. \& Batty, G. D. Sex hormones and cause-specific mortality in the male veterans: The Vietnam Experience Study. QJM Mon. J. Assoc. Phys. 105(3), 241-246 (2012).

17. Nieschlag, E., Kramer, U. \& Nieschlag, S. Androgens shorten the longevity of women: Sopranos last longer. Exp. Clin. Endocrinol. Diabetes 111(4), 230-231 (2003).

18. Gagnon, S. S. et al. Basal endogenous steroid hormones, sex hormone-binding globulin, physical fitness, and health risk factors in young adult men. Front. Physiol. 9, 1005 (2018).

19. Yeap, B. B. Hormones and health outcomes in aging men. Exp. Gerontol. 48(7), 677-681 (2013).

20. Haring, R. et al. Age-specific reference ranges for serum testosterone and androstenedione concentrations in women measured by liquid chromatography-tandem mass spectrometry. J. Clin. Endocrinol. Metab. 97(2), 408-415 (2012).

21. NCT00612742. (2008). Safety and efficacy of LibiGel ${ }^{\triangleright}$ for treatment of hypoactive sexual desire disorder in postmenopausal women (BLOOM). ClinicalTrialsgov. (https://clinicaltrials.gov/ct2/home: US National Library of Medicine).

22. Basaria, S. et al. Adverse events associated with testosterone administration. N. Engl. J. Med. 363(2), 109-122 (2010).

23. NCT003518034. (2018). A study to evaluate the effect of testosterone replacement therapy (TRT) on the incidence of major adverse cardiovascular events MACE) and efficacy measures in hypogonadal men (TRAVERSE). ClinicalTrialsgov. (https://clinicaltrials. gov/ct2/show/NCT03518034: US National Library of Medicine).

24. Brooks, R. C. \& Garratt, M. G. Life history evolution, reproduction, and the origins of sex-dependent aging and longevity. Ann. N. Y. Acad. Sci. 1389(1), 92-107 (2017).

25. Stearns, S. C. Issues in evolutionary medicine. Am. J. Hum. Biol. 17(2), 131-140 (2005).

26. Wells, J. C. K., Nesse, R. M., Sear, R., Johnstone, R. A. \& Stearns, S. C. Evolutionary public health: Introducing the concept. Lancet (London, England) 390(10093), 500-509 (2017).

27. Smith, G. D. \& Ebrahim, S. "Mendelian randomization": Can genetic epidemiology contribute to understanding environmental determinants of disease?. Int. J. Epidemiol. 32(1), 1-22 (2003). 
28. Ruth, K. S. et al. Using human genetics to understand the disease impacts of testosterone in men and women. Nat. Med. 26(2), $252-258(2020)$.

29. Larsson, S. C. et al. Genetic predisposition to smoking in relation to 14 cardiovascular diseases. Eur. Heart J. 41, 3304-3310 (2020).

30. Ravindran, R. D. et al. Genetic variants in a sodium-dependent vitamin C transporter gene and age-related cataract. Br. J. Ophthalmol. 103(9), 1223-1227 (2019).

31. Heijmans, B. T., Westendorp, R. G. \& Slagboom, P. E. Common gene variants, mortality and extreme longevity in humans. Exp. Gerontol. 35(6-7), 865-877 (2000).

32. Glymour, M. M. Using Causal Diagrams to Understand Common Problems in Social Epidemiology (Jossey-Bass, 2006).

33. MacLaughlin, E. J. \& Ernst, M. E. From clinical trials to bedside: the use of antihypertensives in aged individuals. Part 1: Evaluation and evidence of treatment benefit. Curr. Hypertens. Rep. 21(11), 82 (2019).

34. Lewis, S. J. \& Brunner, E. J. Methodological problems in genetic association studies of longevity-The apolipoprotein E gene as an example. Int. J. Epidemiol. 33(5), 962-970 (2004).

35. Jha, P. et al. 21st-century hazards of smoking and benefits of cessation in the United States. N. Engl. J. Med. 368(4), 341-350 (2013).

36. Bjelakovic, G., Nikolova, D., Gluud, L. L., Simonetti, R. G. \& Gluud, C. Antioxidant supplements for prevention of mortality in healthy participants and patients with various diseases. Cochrane Database Syst. Rev. 3, Cd007176 (2012).

37. Schooling, C. M. et al. Genetic predictors of testosterone and their associations with cardiovascular disease and risk factors: A Mendelian randomization investigation. Int. J. Cardiol. 267, 171-176 (2018).

38. Luo, S., Au Yeung, S. L., Zhao, J. V., Burgess, S. \& Schooling, C. M. Association of genetically predicted testosterone with thromboembolism, heart failure, and myocardial infarction: Mendelian randomisation study in UK Biobank. BMJ 364, 1476 (2019).

39. Budoff, M. J. et al. Testosterone treatment and coronary artery plaque volume in older men with low testosterone. JAMA 317(7), 708-716 (2017).

40. Ajayi, A. A. \& Halushka, P. V. Castration reduces platelet thromboxane A2 receptor density and aggregability. QJM Mon. J. Assoc. Phys. 98(5), 349-356 (2005).

41. Herman, S. M. et al. Androgen deprivation is associated with enhanced endothelium-dependent dilatation in adult men. Arterioscler. Thromb. Vasc. Biol. 17(10), 2004-2009 (1997).

42. McCredie, R. J. et al. Vascular reactivity is impaired in genetic females taking high-dose androgens. J. Am. Coll. Cardiol. 32(5), 1331-1335 (1998).

43. van Kesteren, P. J. et al. The effects of sex steroids on plasma levels of marker proteins of endothelial cell functioning. Thromb. Haemost. 79(5), 1029-1033 (1998).

44. Jaillon, S., Berthenet, K. \& Garlanda, C. Sexual dimorphism in innate immunity. Clin. Rev. Allergy Immunol. 56(3), 308-321 (2019).

45. Schooling, C. M. \& Zhao, J. V. Strengthening the immune system for cancer prevention. Proc. Natl. Acad. Sci. U.S.A. 115(19), E4316-E4317 (2018).

46. Wu, Y. et al. Single dose testosterone administration increases impulsivity in the intertemporal choice task among healthy males. Horm. Behav. 118, $104634(2020)$

47. Gardner-Thorpe, D., O’Hagen, C., Young, I. \& Lewis, S. J. Dietary supplements of soya flour lower serum testosterone concentrations and improve markers of oxidative stress in men. Eur. J. Clin. Nutr. 57(1), 100-106 (2003).

48. Wang, C. et al. Low-fat high-fiber diet decreased serum and urine androgens in men. J. Clin. Endocrinol. Metab. 90(6), 3550-3559 (2005).

49. Habito, R. C. \& Ball, M. J. Postprandial changes in sex hormones after meals of different composition. Metabolism 50(5), 505-511 (2001).

50. Berrino, F. et al. Reducing bioavailable sex hormones through a comprehensive change in diet: The diet and androgens (DIANA) randomized trial. Cancer Epidemiol. Biomarkers Prev. 10(1), 25-33 (2001).

51. van Gemert, W. A. et al. Effect of weight loss, with or without exercise, on body composition and sex hormones in postmenopausal women: The SHAPE-2 trial. Breast Cancer Res. 17(1), 120 (2015).

52. Haqq, L., McFarlane, J., Dieberg, G. \& Smart, N. Effect of lifestyle intervention on the reproductive endocrine profile in women with polycystic ovarian syndrome: A systematic review and meta-analysis. Endocr. Connect. 3(1), 36-46 (2014).

53. Campbell, K. L. et al. Reduced-calorie dietary weight loss, exercise, and sex hormones in postmenopausal women: Randomized controlled trial. J. Clin. Oncol. 30(19), 2314-2326 (2012).

54. Moran, L. J. et al. Long-term effects of a randomised controlled trial comparing high protein or high carbohydrate weight loss diets on testosterone, SHBG, erectile and urinary function in overweight and obese men. PLoS ONE 11(9), e0161297 (2016).

55. Hayes, L. D. \& Elliott, B. T. Short-term exercise training inconsistently influences basal testosterone in older men: A systematic review and meta-analysis. Front. Physiol. 9, 1878 (2018).

56. Muller, M. J. et al. Metabolic adaptation to caloric restriction and subsequent refeeding: The Minnesota Starvation Experiment revisited. Am. J. Clin. Nutr. 102(4), 807-819 (2015).

57. Schooling, C. M., Au Yeung, S. L., Freeman, G. \& Cowling, B. J. The effect of statins on testosterone in men and women, a systematic review and meta-analysis of randomized controlled trials. BMC Med. 11, 57 (2013).

58. Barba, M. et al. The effects of metformin on endogenous androgens and SHBG in women: A systematic review and meta-analysis. Clin. Endocrinol. 70(5), 661-670 (2009).

59. Casulari, L. A., Caldas, A. D., Domingues Casulari Motta, L. \& Lofrano-Porto, A. Effects of metformin and short-term lifestyle modification on the improvement of male hypogonadism associated with metabolic syndrome. Minerva Endocrinol. 35(3), 145-151 (2010).

60. Conte, D. et al. Aspirin inhibits androgen response to chorionic gonadotropin in humans. Am. J. Physiol. 277(6), E1032-1037 (1999).

61. Vanky, E., Salvesen, K. A. \& Carlsen, S. M. Six-month treatment with low-dose dexamethasone further reduces androgen levels in PCOS women treated with diet and lifestyle advice, and metformin. Hum. Reprod. 19(3), 529-533 (2004).

62. Doerr, P. \& Pirke, K. M. Dexamethasone-induced suppression of the circadian rhythm of plasma testosterone in normal adult males. J. Steroid Biochem. 10(1), 81-86 (1979).

63. Burgess, S., Davies, N. M. \& Thompson, S. G. Bias due to participant overlap in two-sample Mendelian randomization. Genet. Epidemiol. 40(7), 597-608 (2016).

64. Lopez, P. M., Subramanian, S. V. \& Schooling, C. M. Effect measure modification conceptualized using selection diagrams as mediation by mechanisms of varying population-level relevance. J. Clin. Epidemiol. 113, 123-128 (2019).

65. Collins, R. What makes UK Biobank special?. Lancet (London, England) 379(9822), 1173-1174 (2012).

66. Bowden, J. et al. Assessing the suitability of summary data for two-sample Mendelian randomization analyses using MR-Egger regression: The role of the I2 statistic. Int. J. Epidemiol. 45(6), 1961-1974 (2016).

67. Slob, E. A. W. \& Burgess, S. A comparison of robust Mendelian randomization methods using summary data. Genet. Epidemiol. 45, $131(2020)$.

68. Schooling, C. M., Zhao, J. V., Au Yeung, S. L. \& Leung, G. M. Investigating pleiotropic effects of statins on ischemic heart disease in the UK Biobank using Mendelian randomisation. Elife 9, e58567 (2020).

69. Keevil, B. G. \& Adaway, J. Assessment of free testosterone concentration. J. Steroid Biochem. Mol. Biol. 190, 207-211 (2019).

70. Zohdy, S. et al. Teeth Sex and Testosterone: Aging in the World's Smallest Primate. PLoS ONE 9(10), e109528. https://doi.org/10. 1371/journal.pone.0109528 (2014). 


\section{Author contributions}

C.M.S. conceptualized the study, conducted the initial analysis and wrote the first draft. J.V.Z. contributed to the conceptualization, extracted data, checked the analysis and reviewed the interpretation.

\section{Funding}

This research received no specific grant from any funding agency in the public, commercial or not-for-profit sectors.

\section{Competing interests}

The authors declare no competing interests.

\section{Additional information}

Supplementary Information The online version contains supplementary material available at https://doi.org/ 10.1038/s41598-021-93360-z.

Correspondence and requests for materials should be addressed to C.M.S.

Reprints and permissions information is available at www.nature.com/reprints.

Publisher's note Springer Nature remains neutral with regard to jurisdictional claims in published maps and institutional affiliations.

(c) (i) Open Access This article is licensed under a Creative Commons Attribution 4.0 International License, which permits use, sharing, adaptation, distribution and reproduction in any medium or format, as long as you give appropriate credit to the original author(s) and the source, provide a link to the Creative Commons licence, and indicate if changes were made. The images or other third party material in this article are included in the article's Creative Commons licence, unless indicated otherwise in a credit line to the material. If material is not included in the article's Creative Commons licence and your intended use is not permitted by statutory regulation or exceeds the permitted use, you will need to obtain permission directly from the copyright holder. To view a copy of this licence, visit http://creativecommons.org/licenses/by/4.0/.

(C) The Author(s) 2021 